Recibido: julio de 2017

Aprobado: agosto de 2017

DOI: https://doi.org//0.15332/rev.m.v14i0.2174

Viviendas campesinas municipio de Onzaga, Santander Fuente: Sandra Marcela Muñoz Zea, 2017.

Trabajo de investigación adscrito a la Línea de Investigación en Planificación y Gestión del Territorio del Grupo de Investigaciones de la Facultad de Arquitectura de la Universidad Santo Tomás Seccional Bucaramanga (GINVEARQUI). Líder de línea: Carlos Humberto Gómez Arciniegas, Arquitecto por la Universidad Santo Tomás Seccional Bucaramanga, MSc Lancaster University (Reino Unido) PhD Politécnico di Milano (Italia). Correo electrónico: carlos.gomez@ustabuca.edu.co

* Médica veterinaria y zootecnista de la Universidad de Caldas, especialista en Gestión de Proyectos Sociales por la Universidad Autónoma de Manizales, magíster en Desarrollo Rural por la Universidad Nacional de Costa Rica y candidata a magíster en Ordenamiento Territorial por la Universidad Santo Tomás. Integrante del grupo de investigación USTAGRI- Facultad de Administración de Empresas Agropecuarias - Universidad Santo Tomás. Correo electrónico: sandrazea72@gmail.com

\section{EL PAISAJE AGROPECUARIO COMO REFERENTE PARA EL ORDENAMIENTO DEL TERRITORIO RURAL. UNA APROXIMACIÓN AL CASO DEL MUNICIPIO DE ONZAGA, SANTANDER*}

Sandra Marcela Muñoz Zea** - Universidad Santo Tomás, Colombia

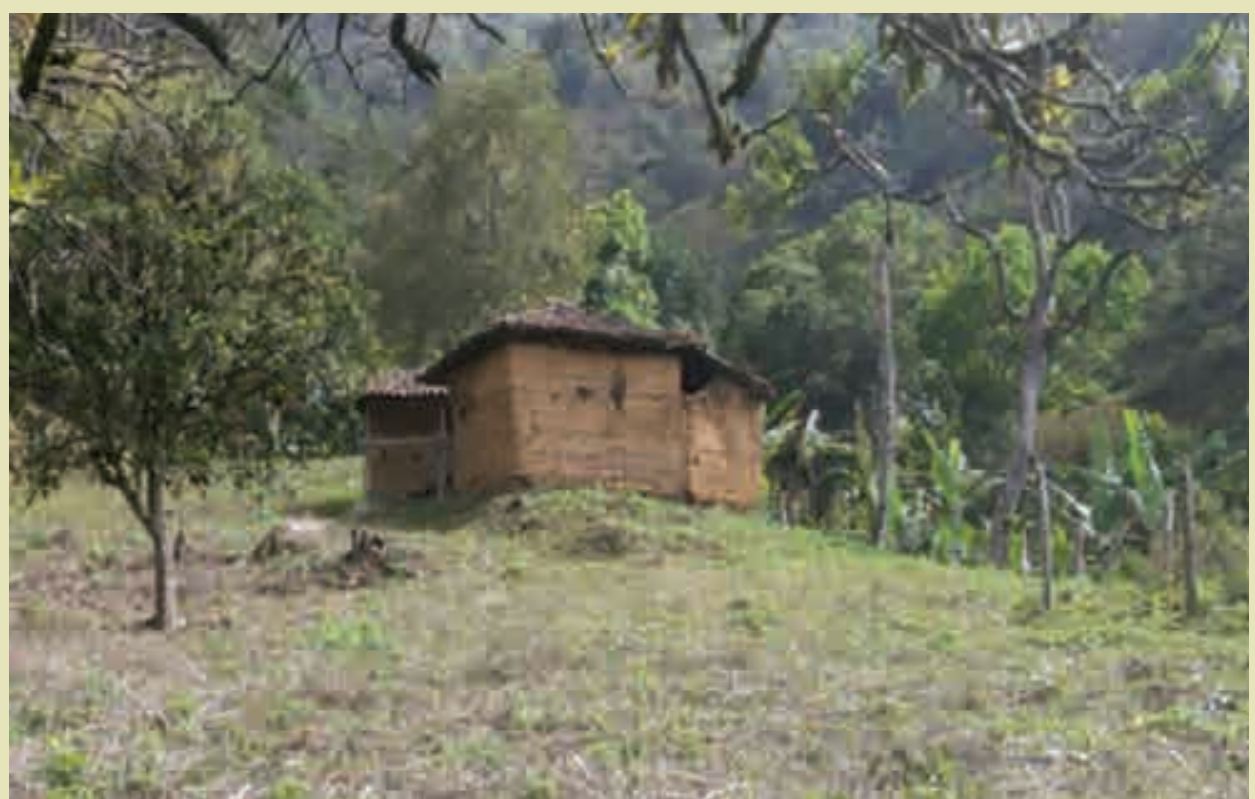

\section{RESUMEN}

El presente artículo centra su atención en la importancia del concepto de los paisajes agrarios como herramienta de diagnóstico que contribuye a la formulación de acciones futuras para el ordenamiento territorial rural en muchas regiones de Colombia. Se parte, por tanto, de una aproximación a la normativa colombiana, específicamente en lo concerniente a la aprehensión del territorio rural, evidenciándose un desconocimiento gubernamental sobre la complejidad del componente territorial. Posteriormente, se desarrolla un ejercicio de análisis con base en experiencias y avances académicos realizados especialmente en Europa, donde se asumen las unidades productivas agropecuarias (UPA) como referentes de la expresión paisajística agraria, dada la complejidad de su estructura, de su conformación, así como su injerencia sobre el paisaje que se establece. Sucesivamente, se explora el concepto de "paisaje" en la planificación del territorio, ligado en particular al paisaje agrario y a sus connotaciones. Por tanto, se acude también a estudio de casos, los cuales contribuyen a crear puntos de reflexión para el conocimiento del paisaje agrario a partir de buenas prácticas documentadas a través de la investigación. En la parte conclusiva, se fortifica el concepto de unidades productivas agropecuarias (UPA) en la interpretación del territorio, lo cual abre un derrotero hacia la identificación de las características paisajísticas naturales del municipio de Onzaga. Finalmente, se articulan las debidas conclusiones del estudio presentado en el artículo, las cuales dejan claro la intención de crear una mirada prospectiva sobre dicho territorio.

\section{PALABRAS CLAVE}

Ordenamiento territorial, paisaje agrario, unidades productivas agropecuarias, Onzaga. 


\section{THE AGRICULTURAL LANDSCAPE AS A REFERENCE FOR THE ORDER OF THE RURAL TERRITORY. AN APPROXIMATION TO THE CASE OF THE MUNICIPALITY OF ONZAGA, SANTANDER}

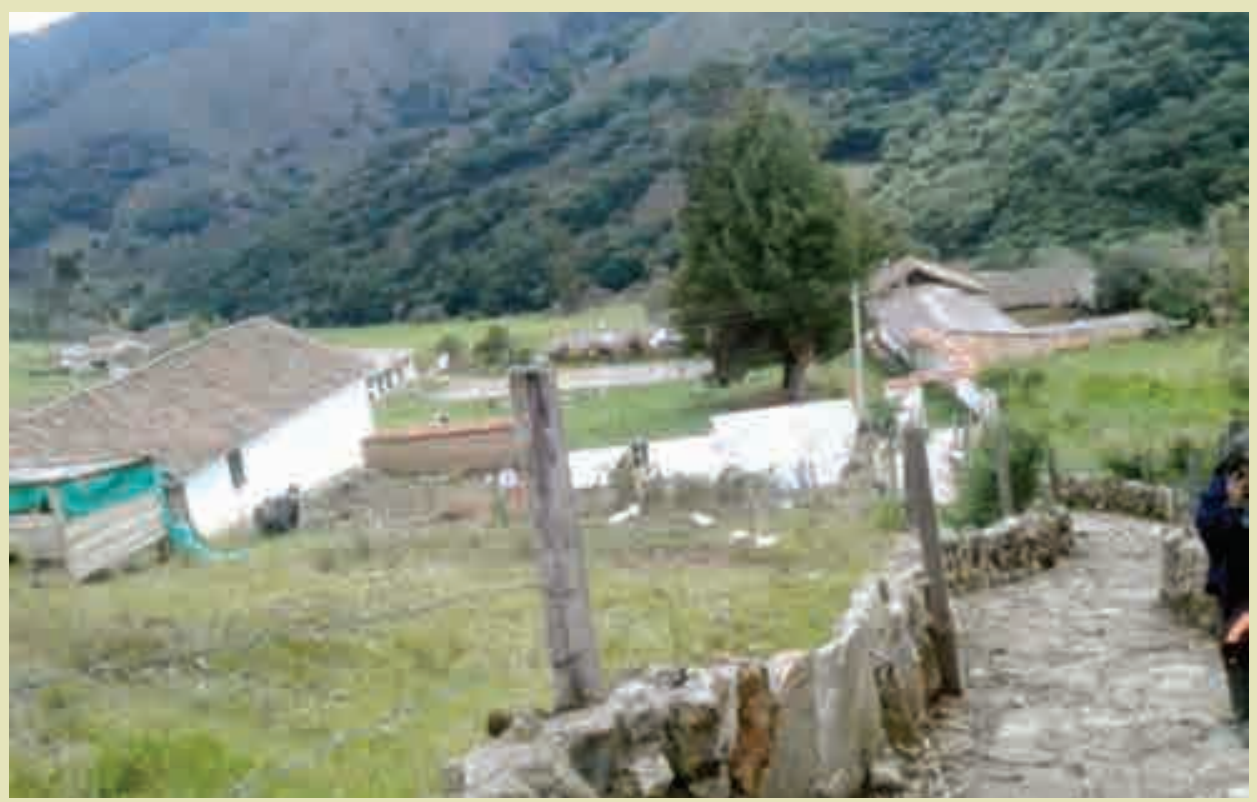

Paisaje rural del municipio de Onzaga, Santander Fuente: Sandra Marcela Muñoz Zea, 2017.

\section{ABSTRACT}

This paper focuses on the concept of agricultural landscapes as an important diagnostic tool which allows to shape future actions linked to rural land-use planning within regions in Colombia. Therefore, an approach to Colombian legislation is initiated, specifically with regard to the apprehension of rural territory, evidencing a lack of government knowledge about the complexity of the territorial component. Afterwards, an analytical exercise based on experiences and academic advance, mainly Europeans, is major carried out since there agricultural productive units are assumed as referents of the agrarian landscape expression, given the complexity of its structure, its conformation, as well as its interference on the landscape that is established. Subsequently, the concept of "landscape" in land planning matters is explored, linked in particular to the agrarian landscape and its connotations. Therefore, case studies arem taken into account, since them contribute to create several reflection points for the knowledge of the agrarian landscape, based on best practices documented through research. In concluding, the concept of agricultural productive units (UPA) for the interpretation of the territory is strengthened, which opens a path towards the identification of the natural landscape characteristics of the municipality of Onzaga. Finally, the proper conclusions of the study presented in the article are structured, making clear the intention to create a prospective look over that territory.

\section{KEYWORDS}

Land management, agricultural landscape, farming productive units, Onzaga. 


\section{INTRODUCCIÓN}

El Departamento Nacional de Planeación de Colombia - DNP ha clasificado el 84,7\% de los municipios del país como áreas rurales en las cuales se aloja el 30,4\% de la población total del país (DNP, 2015). En esta área se promueve una economía dependiente en alto grado, más del $70 \%$ de acuerdo con la misma fuente, de actividades primarias como la agricultura familiar y la explotación de minas y canteras a pequeña escala que sostienen buena parte de los ingresos de la mayoría de los habitantes mencionados. A pesar de que la población rural en Colombia ha venido disminuyendo, siguen siendo evidentes las enormes brechas de desarrollo social que sufre el entorno rural colombiano. Por ejemplo, el municipio de Onzaga, principal escenario aplicativo de este estudio, ha sufrido un descenso en su población entre el 2005 y 2016, pasando de 5.707 a 5.003 habitantes, donde un $79 \%$ de dicha población es considerada rural y un $21 \%$ urbana. Este fenómeno puede explicarse en parte, a partir de las cifras entregadas por el Índice de Necesidades Básicas Insatisfechas (NBI), modalidad de medición de la pobreza, recomendada por la Comisión Económica para América Latina y el Caribe (CEPAL), la cual identifica las carencias familiares en términos de cuatro ámbitos específicos: ingresos, educación, servicios básicos y vivienda. Los datos presentados para el municipio de Onzaga son preocupantes especialmente para el sector rural donde el nivel de pobreza llega al $64.16 \%$ frente al $13.17 \%$ en la cabecera municipal, según el censo del DANE (2005). Lo anterior se evidencia aun teniendo en cuenta que sus riquezas naturales permiten a los habitantes suplementar una buena cantidad de elementos de la canasta básica familiar; los demás factores necesarios para cubrir las necesidades básicas no se alcanzan con los bajos niveles de ingresos que tienen los pobladores como se evidenciará más adelante.

Por esta, entre muchas otras razones, el desarrollo de la ruralidad colombiana, basada entre otras en las actividades ya mencionadas, requiere del soporte estatal pertinente. A esto se suma la inoperatividad de la planificación estatal para el sector rural y la generalización que caracteriza la mayoría de los estudios sobre este territorio, así como la normativa emitida, ya sea a nivel nacional, departamental o municipal. En este último rango, los municipios con una fuerte vocación agrícola y pecuaria no están oportunamente salvaguardados por una normativa que promueva el desarrollo sostenible del territorio. Instrumentos como los Esquemas de Ordenamiento Territorial - EOT son en diversas ocasiones un acopio mal estructurado de otros documentos e inclusive producto de una falsa lectura e interpretación del territorio.

A la luz de las anteriores consecuencias, este artículo se presenta como un llamado a la conciencia de estudiosos y planificadores para adoptar una posición más firme sobre la importancia que el territorio rural merece $y$, por ende, la meticulosidad que demanda el estudio de los subsistemas que lo conforman y lo caracterizan. En consecuencia, el documento aborda la ya nota problemática del agro colombiano desde la dimensión normativa y las principales lagunas e inconsistencias que se están presentando en términos de plano ficción y gestión, haciendo hincapié en las debilidades actuales al momento de leer adecuadamente el territorio rural. Sucesivamente, se explora sobre el concepto de "paisaje" en la planificación del territorio, ligado en particular al paisaje agrícola y pecuario, dado que sin duda el territorio rural conforma un escenario que debe ser adecuadamente protegido y planificado. Por tanto, se acude también a estudio de casos provenientes de otros ámbitos en los cuales se han llevado a cabo buenas prácticas (best practices) que coadyuvan a crear puntos de reflexión para el conocimiento del paisaje agropecuario. Este paso conlleva abordar el concepto de unidades productivas agropecuarias - UPA, símil implícito de las tradicionales "fincas" colombianas, como instrumento de gran utilidad en la interpretación y planificación del territorio. El proceso esbozado hasta el momento traza una trayectoria hacia la identificación de las características paisajísticas naturales del municipio de Onzaga, dejando sobre la mesa una mirada prospectiva sobre dicho municipio y, por tanto, sobre el estudio detallado de sus características 
físicas, sociales y económicas, a partir del concepto de las UPA, las cuales contribuirán a una correcta lectura del territorio así como a la formulación precisa de los relativos instrumentos de planificación del mismo.

\section{LA NORMATIVA COLOMBIANA Y LA LECTURA DEL TERRITORIO RURAL}

Las políticas públicas concernientes al desarrollo de los territorios rurales como la 152 de 1994, Ley Orgánica del Plan de Desarrollo y la Ley 388 de 1997, Ley de Desarrollo Territorial; disponen los procesos e instrumentos para la planificación y la gestión municipal como elementos de orientación a la selección, ordenamiento y orientación de las actividades que se emplazan en una localidad, especificando además los alcances de las mismas. En lo referente al ordenamiento, esta labor se realiza a través de los Planes de Ordenamiento Territorial - POT; los Planes Básicos de Ordenamiento Territorial - PBOT y los Esquemas de Ordenamiento Territorial - EOT; la clasificación de dichos instrumentos se establece de acuerdo con el número de habitantes de la localidad en estudio, es decir, POT para poblaciones mayores a 100.000 habitantes, PBOT de 30.000 a 100.000 habitantes y EOT para menores a 30.000 habitantes.

Aquí es válido recordar las anotaciones de Agudelo (2006) al respecto de los desajustes de la ley frente a las características actuales de los territorios rurales en Colombia. En primer lugar, indica Agudelo, está la realidad estadística que no se identifica con la distribución que hace la ley, ya que de los 1 . I 14 municipios del país, más del $75 \%$ corresponde a localidades con menos de 15.000 personas. Lo segundo, es que las exigencias para el ordenamiento, tanto rural como urbano, van disminuyendo en la medida que disminuye el número de habitantes, ello es, la rigurosidad de la ley y la pertinencia de los instrumentos es proporcional a la disminución en el número de habitantes; la disciplina con la que se ordenan las acciones del suelo rural, menos denso, es poco exigente con respecto a la de los espacios urbanos; $y$ lo tercero es el origen de la Ley que toma referentes de la Ley del Suelo española que además de responder a características rurales evidentemente diferentes, tiene un marco legal que faculta a las localidades de manera distinta a como se propone en Colombia. De hecho, en España, los municipios tienen muy pocas competencias en materia de planificación del suelo rural, a no ser que se trate de suelo rural urbanizable; asuntos como las restricciones de uso (suelo rural de protección) y la promoción de actividades productivas, son a menudo de competencia provincial, autonómica y hasta comunitaria (Agudelo, 2006), en el caso de Colombia, asunto de injerencia departamental.

Con lo anterior se hacen evidentes las debilidades de la Ley 388 de 1997 para comprender el contexto de los territorios rurales colombianos y en consecuencia la poca pertinencia y eficiencia de su desempeño. Buena parte de las razones de esta descontextualización nacen desde el concepto mismo que la ley tiene de la ruralidad. En el documento no hay una postura clara sobre el tema y por ello las disposiciones se hacen de manera generalizada, sin consideración alguna a las particularidades de las comunidades y territorios rurales que en el documento se atienen de manera indistinta a las urbanas. Las únicas disposiciones diferenciales se refieren a la identificación de usos del suelo circundante a la mancha urbana: suelo de expansión, suelo suburbano y el rural disperso, de manera que el ordenamiento se construye en función de las ciudades, con la preponderancia de lo urbano y bajo el concepto de lo rural como subsidiario a aquello.

En dicha Ley, el continuo del suelo suburbano se identifica como rural disperso, aquí la conexión de utilidad con las ciudades se desvanece y la ley pareciera terminar sus deberes; las funciones del suelo y las identidades particulares de las localidades se homogenizan y por

M 83 REVISTA M VOL. 14 ENERO - DICIEMBRE 2017 • FACULTAD DE ARQUITECTURA • UNIVERSIDAD SANTO TOMÁS COLOMBIA 
defecto se relacionan con las actividades agropecuarias o de protección ambiental como únicas actividades económicas reconocidas. He aquí otra falta de atención a la realidad. Hoy, la multiplicidad de actividades económico-productivas en la ruralidad es evidente, ocupaciones como el turismo, la explotación minera, la agroindustria, la actividad comercial, entre otros, complejizan los usos del suelo y dotan al territorio de nuevas características, por lo que es de vital importancia ofrecerles un manejo diferencial amparando normativamente sus exigencias de manera que ellas se liberen del tratamiento informal y anónimo que se les da en la normativa actual.

Ahora, es necesario anotar que la ley sí propone herramientas para la lectura del territorio. En el artículo I I 2 de la Ley 388 se dispone el Expediente Municipal (también llamado Expediente Urbano), que se define como el

Sistema de información que permite evaluar la aplicación de sus estrategias e instrumentos de gestión (de la Ley 388), a la vez que se convierte en la herramienta técnica con la que se puedan soportar las modificaciones y ajustes al POT cuando se ha iniciado el proceso de su revisión, como se consagra en el artículo 28 de la Ley 388 de 1997 y en el Decreto 932 de 2002 (Minambiente, 2004).

La función misional del expediente es entonces identificar las características, vocaciones de uso, formas de tenencia, nivel de ocupación y en general el acervo de particularidades que dan sentido a la planificación y la gestión de los suelos municipales.

De acuerdo con la guía metodológica del expediente urbano formulado por el Ministerio de Ambiente, Vivienda y Desarrollo Territorial - Minambiente (2004), la primera etapa en el proceso de creación del expediente es la caracterización y diagnóstico territorial. El valor de este diagnóstico estriba principalmente en su capacidad para dilucidar los cambios y dinámicas que en el tiempo vive el territorio, lo que explica que a partir de él se construyan las bases de la planificación con pertinencia, sin embargo, pormenorizar este proceso se ha convertido en una práctica general, llevándolo incluso a la omisión de su elaboración o la burda copia de los elaborados con anterioridad. Con el artículo I I 2 la Ley sí reconoce el papel fundamental del análisis previo a la planificación, sin embargo, no es clara en sus alcances y más que un método sugiere pasos para su elaboración sin proponer un corpus teórico y metodológico orientador.

No muy ajenos a estas complicaciones y en un intento por resolver situaciones similares, varios gobiernos europeos vienen desarrollando investigaciones sobre métodos y herramientas que viabilicen la labor del ordenamiento territorial en clave de complejidad. Es así como surge el interés por apropiar la experiencia europea en cuanto a la valoración del paisaje y al empleo del mismo como herramienta de ordenamiento y planificación. El aporte de las investigaciones referentes al reconocimiento y valoración del patrimonio paisajístico de los territorios no se limita a la identificación y descripción de estos espacios para objetivos académicos. Su contribución aplicativa comienza a considerarse particularmente en la generación de los planes y herramientas de ordenamiento territorial en virtud de la complejidad y dinamismo que implica su abordaje. Además, el uso de este elemento territorial como referente de gestión, es útil para localidades con recursos económicos y de equipo humano limitados, precisamente porque su abordaje requiere una lectura integral, presente y sincrónica de todas las dimensiones participantes, lo que favorece el uso eficiente de los escasos recursos públicos de municipios como el de Onzaga en el departamento de Santander (Colombia), del cual se hará referencia más adelante.

Como lo refiere Zoido (1999), el paisaje puede aportar en varios sentidos a la instrumentalización del ordenamiento: en primer lugar, muy relativo a la necesidad de los diagnósticos antes mencionados, el paisaje tiene la capacidad de mostrar los aciertos y disfunciones del uso 
del suelo ayudando a comprender sus formas y causas; en segundo lugar, contribuye a corregir las disfunciones en la ubicación y disposición de los elementos en el territorio. En tercer lugar, el enfoque paisajístico propone la inclusión de las personas que finalmente son las que aprueban toda propuesta de ordenamiento y planificación. En este último sentido se entiende también por qué la participación de la comunidad es vital. La lectura de la comunidad es la única con idoneidad para referenciar todo el conocimiento histórico y presente de la conformación de aquellas dimensiones. Hacer partícipe a la población cumple además con una de las exigencias principales que el Departamento Nacional de Planeación de Colombia - DNP solicita dentro de los procesos de planificación, y que está vinculada a los principios de equidad e inclusión.

El ejercicio comunitario en la estructura del paisaje se relaciona específicamente con los usos que la sociedad hace del espacio geográfico que habita. En esta relación con los recursos naturales se encuentra la génesis de la dinámica paisajística cuando la acción del hombre está presente y es quizá este paisaje, el constituido por las labores agrarias, uno de los que mejor expresan dichas relaciones en el contexto rural. Como lo indica Gómez Sal (1997) "La conservación de la naturaleza -y en concreto los paisajes agrarios valiososdebe entenderse como una acción positiva -no defensiva-, comprometida en la gestión del territorio y en el desarrollo de la población local".

Con esta justificación se llega finalmente a la unidad de análisis seleccionada para la investigación relacionada en este artículo, leída a partir del concepto de las unidades productivas, a su vez entendida como núcleos que generan de por sí paisaje, productividad y otras dinámicas. En consecuencia, en el contexto rural, el paisaje agrario puede leerse a través de las unidades productivas que modifican el espacio geográfico local.

\section{El concepto de paisaje en la planificación del territorio}

Para Gómez Villarino (20 I 2), el paisaje es la "percepción polisensorial y subjetiva de la expresión externa en que se manifiesta el sistema territorial. En este concepto es interesante el análisis que hace el autor de sus componentes: un objetivo que corresponde al territorio y a la huella (carácter) que imprime la acción humana en él; a este conjunto el autor lo denomina "base paisajística". El componente subjetivo, es lo relativo a la percepción que cada individuo tiene de lo que observa. En esta primera aproximación se percibe la atención que pone el autor en la experiencia sensorial de quien observa y en este sentido el alto contenido de subjetividad del concepto. A partir de esta orientación estética puede decirse que se plantearon las conceptualizaciones iniciales desde disciplinas como la geografía y la arquitectura, justificando la defensa y el resguardo natural y patrimonial de los paisajes con alto valor visual, principalmente.

Luego, con el establecimiento del Convenio Europeo del Paisaje, el concepto de paisaje prácticamente sube su categoría y ahora se asume territorialmente; es decir, se asimila la idea de que todo territorio es un paisaje y cada paisaje es la manifestación particular de un territorio (Convention Européenne du Paysage, 2000). Mata (2008) acuña el término territorialización que resume claramente esta lectura. El término especifica que "cada territorio se manifiesta paisajísticamente en una fisonomía singular y en plurales imágenes sociales que hacen del paisaje un aspecto importante de la calidad de vida de la población". Gómez Orea también aporta al sentido del territorio, identificando el "sistema territorial como una construcción social sobre el sustrato natural, muchos de cuyos elementos permanecen" (Gómez, Gómez, \& Gómez, 2012) y explica que el sistema se sostiene en tres elementos básicos: la estructura, el funcionamiento y la imagen.

M 85 REVISTA M VOL. 14 ENERO - DICIEMBRE 2017 - FACULTAD DE ARQUITECTURA • UNIVERSIDAD SANTO tOMÁS COLOMBIA 
Esta lectura compleja del paisaje es el primer concepto que sirve como referente para esta investigación, básicamente porque como complementan Cano (2006) y Mata (2008) entre otros, el mismo hecho de la territorialización plantea que las intervenciones de las poblaciones sobre el medio natural (urbanización, actividad agropecuaria, industrial, etc.) deben ser planeadas, ordenadas y gestionadas con el consenso de las comunidades que, como se evidenció antes, constituyen el marco social que expresa un paisaje. En consecuencia, la determinación jurídica del paisaje como herramienta de gestión y ordenamiento se justifica lógicamente en su virtud como proyección de la calidad de vida de las comunidades que lo construyen.

De acuerdo con autores como Mata (2008) y Gómez (20I2), el paisaje es un concepto indeterminado y polisémico que en su lectura básica y unidimensional se refiere a la percepción polisensorial que un individuo tiene sobre el espacio geográfico que observa. Sin embargo, a partir del incremento en los últimos años de los interesados en el tema, ahora se le propone como una unidad sistémica, integrada y complementaria que al menos contempla al territorio, la sociedad y el patrimonio en la base de aquella percepción visual. (Gómez Villarino, 2012); (Cano, 2006); (Irastrorza, 2006); (Mata, 2008); (Gómez \& Riesco, 20I0). En este sentido el Convenio Europeo del Paisaje en el 2000 lo definió como: "cualquier parte del territorio, tal y como es percibida por las poblaciones, cuyo carácter resulta de la acción de los factores naturales y humanos y de sus interrelaciones". Esta lectura compleja le dio un nuevo sentido al paisaje y lo llevó a la categoría de recurso natural indisoluble del territorio en el que subyace; $y$ por ende, de la sociedad que da sentido al espacio geográfico. Así, el paisaje puede considerarse como la punta visible de un "iceberg" sostenida por intrincadas relaciones entre los factores económicos, culturales, sociales, naturales y ecosistémicos que lo conforman.

\section{El paisaje agrario y sus connotaciones}

El contexto del desarrollo económico latinoamericano ha estado históricamente ligado a la producción de bienes primarios, especialmente a la producción agropecuaria. En razón a ello, la expresión paisajística del territorio refleja la fuerte interacción de estas actividades con la base geográfica natural. Esto se corresponde con la conceptualización del paisaje agrario que autores como Gómez Sal (1997) acuñan:

La acción humana conduce a un tipo de configuración de los componentes físicos y biológicos de los ecosistemas que, para cada territorio, responde a un compromiso entre la estrategia ideal de aprovechamiento de los recursos y los imperativos del mercado. El resultado son modelos de paisaje que dependen de cada tipo de circunstancias socioeconómicas y ecológicas.

Esta visión del paisaje reconoce el valor de los arreglos productivos que las comunidades hacen de sus espacios en torno al aprovechamiento de los recursos que poseen y a las condiciones ambientales que los determinan. La agricultura es una actividad esencialmente adaptativa que vincula el ingenio, el esfuerzo y la creatividad humana, con una oferta de recursos naturales que las comunidades deben aprovechar de la manera más eficiente y administrar en consideración al carácter finito y de mutabilidad permanente de dichos recursos. Esta serie de condiciones genera "modelos" regionales productivos que la humanidad ha aprendido a admirar, ya sea por su valor eminente paisajístico o reconociendo su valor patrimonial.

En este último sentido la Organización de las Naciones Unidas para la Educación, la Ciencia y la Cultura - UNESCO, se ha dado a la tarea de visibilizar la patrimonialidad de los paisajes agrarios y en primer lugar identifica dos tendencias del concepto. La primera reconoce el valor de lo agrario específicamente por los bienes que la actividad genera: 
(...) el patrimonio agrario es todo aquel legado relacionado con la herencia histórica de la explotación agropecuaria, entendida ésta en un sentido amplio (áreas de cultivo, dehesas, pastizales, plantaciones forestales), bien sea de carácter material (paisajes, edificios relacionados con la producción y la transformación de los productos de la agricultura, infraestructuras y equipamientos agrarios, determinados tipos de hábitat rural) o etnográfico (oficios, artesanías, folklore...) (Silva, 2008).

La segunda orientación, a diferencia de la primera, postula que

La actividad agraria no puede reconocerse y mantenerse en un solo bien, ni siquiera en un conjunto de ellos, sino que se desarrolla en un ámbito territorial. Por esta razón, el procedimiento para identificar y preservar el Patrimonio Agrario debe ser de carácter territorial (Castillo \& Martínez, 20I4).

En este caso, Castillo y Martínez (20I4) ofrecen una visión mucho más integral. La producción agropecuaria no es una acción aislada, sino que hace parte integral de una categoría mayor que es la ruralidad o del modo de vida rural. En este sentido la visión de la acción económico-productiva no es lo único que identifica la ruralidad, sino que la ruralidad es el modo de vida que resulta de la interacción de una comunidad con un territorio en el que los recursos naturales son la base principal de desarrollo. Como lo afirma Echeverri: "Se considera que un territorio es rural cuando el proceso histórico de construcción social que lo define se sustenta principalmente por los recursos naturales y mantiene esta dependencia estructural de articulación" (Echeverri, 20II).

Sin embargo y a pesar de que es clara e ineludible la relación preponderante con los recursos naturales y el medio ambiente, la ruralidad supera la mera actividad agropecuaria e incluye todas las actividades socioeconómicas, culturales, políticas y productivas que pueden desarrollarse en un territorio rural. Es necesario vincular al concepto del paisaje agrario la característica de multifuncionalidad. Este concepto hace referencia al hecho de que hoy en día la funcionalidad de los sistemas económicos rurales no se limita a las actividades agropecuarias, sino que sostiene una diversificación económica con servicios como el turismo, la hostelería, actividades culturales, deportivas y otras actividades de sostenimiento familiar como agroindustria y las artesanías, entre otros. Por esta razón el análisis del paisaje rural, como referente económico de su población debe superar la unidimensionalidad y permitir la vinculación de otros sectores productivos.

\section{El conocimiento del paisaje agrario a través del estudio de caso}

Esta sección resume trabajos de investigación, con diferentes tipos de metodologías y objetivos relacionados con los paisajes agrarios y el patrimonio cultural. Estos trabajos que se referencian hacen interesante el estudio rural $y$, en este caso, a la experiencia de Onzaga porque consideran, por un lado, el análisis del sistema territorial a partir de la idea del paisaje como expresión compleja de la relación del hombre con el área geográfica con la cual se vincula para conformar un territorio particular; y por otro lado, la generación de propuestas de gestión acordes con las condiciones y necesidades de aquellos paisajes, en este caso, los agrarios.

Así bien, el "Plan de manejo para el Paisaje Agavero y las Antiguas Instalaciones Industriales de Tequila", trabajo realizado por Ignacio Gómez Arriola en 2010, describe el proceso de inscripción del paisaje cultural de la comarca del volcán de Tequila en la Lista 
Figura I. Paisaje agavero. Patrimonio de la Humanidad, Jalisco, México

Fuente: www.visitmexico.com/es/actividadesprincipales/tequila/cabalgata-en-el-paisajeagavero

del Patrimonio Mundial de la UNESCO (figura I). Como referente cultural de máximo reconocimiento, el licor Tequila, extraído de la planta de Agave Azul, encierra en su fabricación la expresión del conocimiento y herencia cultural de una región procedente de la comunidad prehispánica Teuchitlán. Esta amplia región se enriquece además con el paisaje natural del volcán de Tequila y el cañón del río de Santiago en el estado mexicano de Jalisco. En estas condiciones, esta dinámica región es un ejemplo visible de la constitución de un paisaje agrario con valor patrimonial y cultural de inmejorable utilidad como eje direccional para la organización de un territorio.

La tradición tequilera que hoy guarda en su mayoría las técnicas productivas, la arquitectura tradicional y el valioso patrimonio industrial mereció el reconocimiento de la UNESCO en razón a tres criterios principales: a) el cultivo ha producido un paisaje distintivo que refleja la fusión entre una tradición productiva local prehispánica con procesos europeos; b) la permanencia de los complejos arquitectónicos e industriales vinculados al proceso productivo del Tequila; y c) El paisaje constituido por campos agaveros, destilerías, haciendas y poblados es un ejemplo excepcional de un asentamiento humano tradicional y un uso de la tierra que es representativo de la cultura específica que se desarrolló en Tequila (Arriola, 20I0).

En respuesta a estas condiciones especiales el Estado mexicano se interesa por preservar su valor cultural y agrario a través de la constitución de un Plan de Manejo en el que los agentes sociales y políticos, responsables de la gestión del territorio, ubiquen su rol y sus responsabilidades en la conservación del mismo. Para este fin, Arriola propuso en primer lugar desarrollar un diagnóstico a fondo de la condición actual del territorio en el que se identifiquen: las problemáticas que eventualmente puedan amenazar la integridad del paisaje agavero; $y$ las condiciones, que al mantenerse, guarden la autenticidad de este. En el proyecto se recogieron los resultados del diagnóstico del Modelo de Ordenamiento Ecológico Territorial del Estado de Jalisco (Gobierno de Jalisco, 1997), que identificó entre otros factores, el deterioro paulatino de la condición de productividad del suelo por manejo inadecuado e insostenible de las técnicas de cultivo; trastornos relacionados con la contaminación ambiental, la deforestación, la pérdida de la biodiversidad y en consecuencia, la ineficiencia productiva de los cultivos que sostienen la economía familiar. Todo ello es una garantía segura de la desaparición del patrimonio cultural agavero, si no se desarrollan acciones que controlen los daños ya causados y prevengan efectos posteriores.

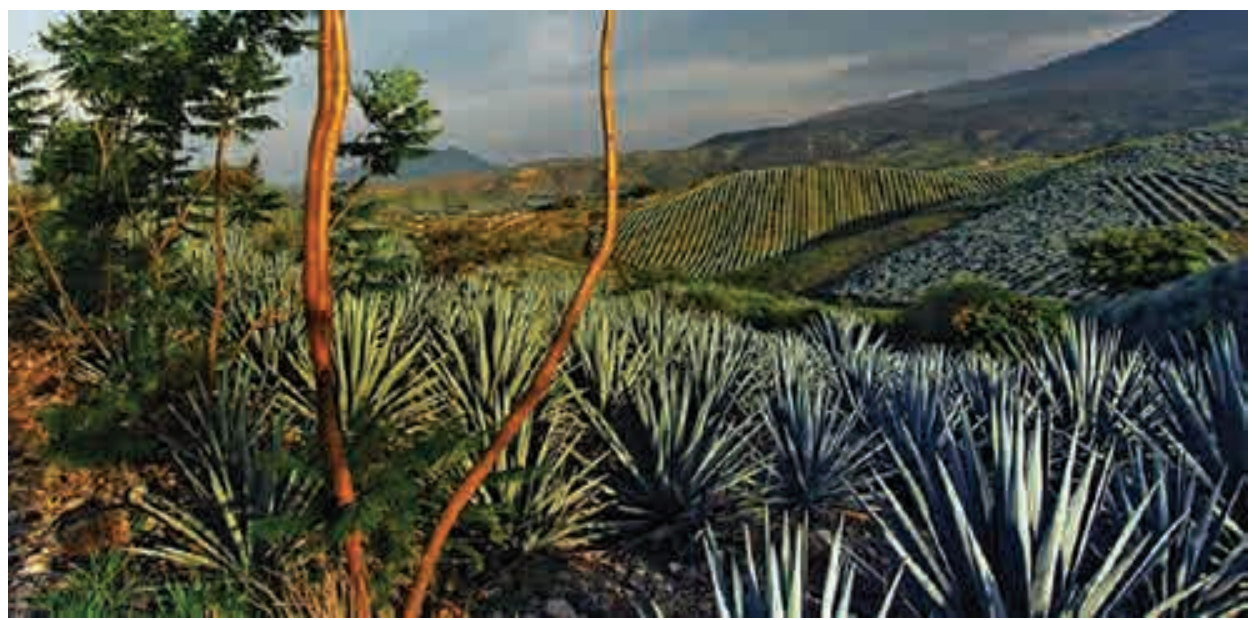

A partir de lo anterior, el proyecto propuso un novedoso instrumento de gestión sin referentes previos en el país, para la planeación, ordenamiento y gestión del territorio del volcán Tequila y su área de influencia: El plan de manejo para el Paisaje Agavero y las Antiguas 
Instalaciones de Tequila. Además, ante la inexistencia de una legislación que amparara el manejo del paisaje agrario y del patrimonio cultural, se trabajó en la creación de estrategias para vincular los contenidos del Plan en los diferentes proyectos de desarrollo urbano y en otros instrumentos locales de gestión. Estas acciones facilitaron la aprobación y adopción de la propuesta. La experiencia en Jalisco da cuenta de la integralidad del aprovechamiento del paisaje, como unidad de gestión y del patrimonial cultural como factor calificador de los procesos de planificación y ordenamiento del territorio.

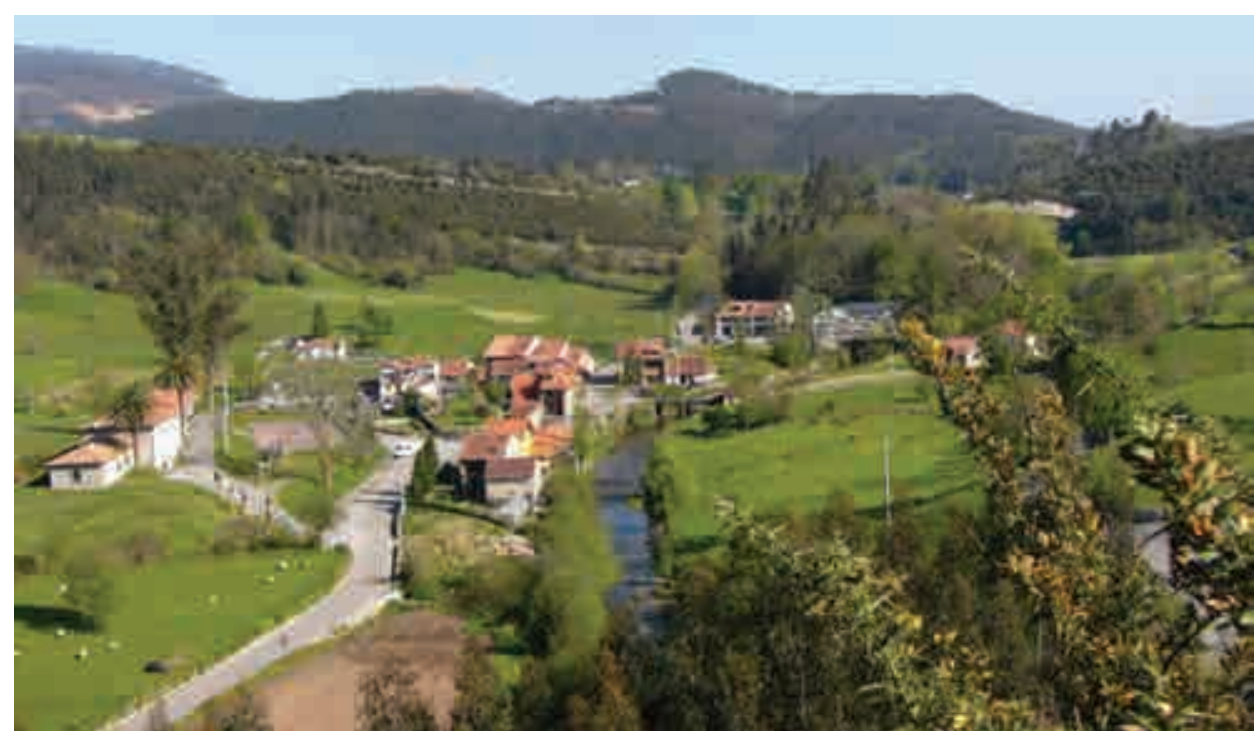

Con la misma misión, hacia la valoración y protección patrimonial, pero con otras orientaciones metodológicas, el trabajo de investigación de Cortijo (20I3) "Paisaje y patrimonio rural: Las "mieses" en Valdáliga, Herrerías y Rionansa” evidencia el valor cultural del paisaje agrario de las "mieses" a través de su caracterización y tipificación en tres municipios del occidente de Cantabria, España. Cabe anotar que las "mieses" son los campos sembrados de cereales en estado de cosecha y cuyas semillas se usan en la elaboración del pan.

Previo a la caracterización el autor hace un interesante recorrido por las orientaciones conceptuales desarrolladas hasta el momento en torno a las diferencias entre el paisaje agrario y el paisaje rural, especialmente con referentes europeos. De este modo, llega a una integración entre los conceptos y las formas de organización de los mismos y, finalmente, propone la orientación de su investigación. El autor identifica en las "mieses" una forma de organización de la agricultura que claramente expresa el patrimonio cultural productivo de la región y conforma un paisaje particular. Por estas razones, son la unidad de análisis de su estudio. Metodológicamente, para la caracterización, el autor propone, básicamente, hacer un trabajo de identificación y diferenciación de las mieses en el territorio del "Soplao" (Cortijo, 20I3) a partir de los siguientes factores, tanto físicos como humanos: a) el tamaño, para diferenciar la agricultura de pequeños y grandes productores; b) la calidad, porque incide en la productividad; c) la ubicación, para establecer diferencias morfológicas; d) las dinámicas y presiones sociales y territoriales que imprimen diferencias paisajísticas en unidades con iguales condiciones físicas; y e) la concentración parcelaria, por los cambios radicales en la morfología parcelaria y la red vial que ella demuestra.

Entre los principales resultados del trabajo el autor reconoce que las mieses tienen un doble componente cultural, pues por un lado son la huella de las acciones territoriales de las sociedades que en épocas pasadas habitaron este espacio y por otro, son el lienzo en el que
Figura 2. Paisaje rural en Valdáliga - Cantabria, España

Fuente: https://www.escapadarural.com/quehacer/valdaliga 
Figura 3. Paisaje agrario en las Islas Canarias, España

Fuente: http://loquesomos.org/por-un-territoriosostenible-canarias-no-es-un-solar/

se plasman los resultados de las políticas territoriales. Así, las mieses se caracterizan por su morfología heredada de la época medieval, pero se diferencian unas de otras, no solo por el tamaño, la calidad o la ubicación que presentan, que son factores físicos; sino también por factores de carácter antrópico y por tanto cultural, que vienen determinados por las acciones territoriales actuales o del pasado (Cortijo, 2013, p. 89). La experiencia de Cantabria es una guía interesante para el análisis del paisaje a partir de la caracterización de unidades de menor tamaño que, en suma, establecen un paisaje reconocido. También valora otros factores como la productividad y el tamaño de las unidades productivas como referentes de la salud del paisaje. En todo caso, se refiere a territorios de valor económico-productivo.

En las islas Canarias, el trabajo de Víctor Martín "Aproximación tipológica a los paisajes agrarios actuales de Canarias" también utiliza una metodología práctica para hacer la descripción de los actuales paisajes agrarios en las islas. El trabajo hace un aporte de interés para diferentes disciplinas. Desde la perspectiva del concepto de paisaje, entendido aquí como la expresión de los elementos que interactúan en un espacio geográfico para dar sentido al territorio, se convierte en un insumo de diagnóstico muy completo para la elaboración de planes de gestión. El trabajo se justifica en que, siendo la actividad agropecuaria la principal fuente de ingresos en el archipiélago, las fuertes transformaciones en las técnicas productivas y en general en la estructura económica del país ha modificado sustancialmente los paisajes agrarios tradicionales (Martín, 2000).

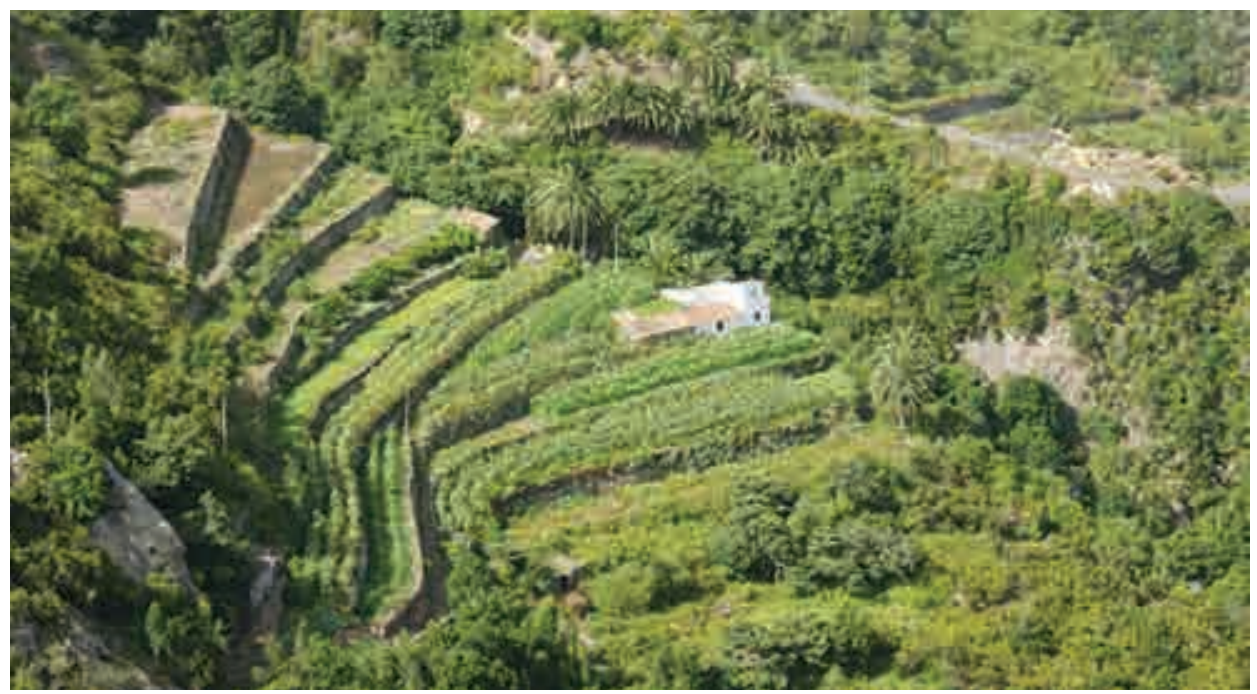

En el mismo sentido del trabajo, Cortijo (20I3) y Martín (2000) clasifican los paisajes agrarios de acuerdo con criterios físico-ecológicos y socio-económicos, pero en este caso, un poco más detallados. Los factores por considerar son: a) la naturaleza del suelo cultivado; b) la localización espacial; c) el sistema de construcción del terrazgo agrícola (pedazo pequeño de tierra para sembrar); d) las características del parcelero productor; e) el régimen hídrico: secano y regadío; f) los tipos de cultivos; g) el destino de la producción agropecuaria; y h) la estructura de la propiedad de la tierra. De acuerdo con estos criterios, la combinación de los mismos y su ubicación en diferentes regiones del archipiélago, el autor concluye en una tipología de los paisajes agrarios integrados en la descripción de quince de ellos.

En otro orden de ideas y con referencia en las modalidades de ordenamiento del territorio agrario, existen innumerables experiencias, especialmente en los países donde 
la participación de la producción agropecuaria y el sector primario de la producción económica siguen siendo primordiales. Para los intereses de la presente investigación es necesario referenciarlas, ya que reconocen la importancia de la gestión del territorio rural y de las tierras de uso agropecuario, considerándolas de manera diferenciada al ordenamiento urbano, como les corresponde, y atendiendo a la alta carga cultural y social de su constitución.

Ahora bien, en el caso de las experiencias locales en Colombia, en el departamento de Antioquia, por una iniciativa de tres municipios del Oriente antioqueño se creó el Distrito Agrario "Valle de San Nicolás" como parte de una de las estrategias del Plan de Ordenamiento Territorial del Altiplano Oriente Antioqueño 1994- 1996. El Distrito es una iniciativa que nace de las preocupaciones por la insostenibilidad económica, social y ambiental de la producción agropecuaria. A esta situación se suma la inexistencia de una política rural consecuente con las condiciones de los pequeños productores y especialmente la debilidad de los métodos para planear los territorios, considerando las fincas como unidad de análisis. El objetivo del Distrito Agrario es el de proteger las economías campesinas e incentivar la producción agroecológica y el mercado justo, promoviendo la soberanía alimentaria, el desarrollo rural sostenible y el bienestar de la población.

Desde 1996 se han desarrollado varios proyectos en el marco del Distrito que le dan vida a las estrategias. En el caso particular del Ordenamiento Territorial, tomando la finca como referente, se usa como método la planificación predial participativa con criterios de sostenibilidad ambiental, social y económica y la construcción de planes de desarrollo rural campesino como modelo de gestión. La idea es que desde la finca se promueva una réplica del modelo que vaya subiendo de categorías territoriales hasta alcanzar el municipio.

Dos son los elementos principales que el proyecto del Distrito Agrario le aporta a la presente investigación; por un lado, la selección de la finca como unidad mínima de planificación con base en su capacidad para reflejar el territorio y, por otro lado, dirigir el interés del ordenamiento hacia la finca como referencia de las actividades económico-productivas y de las características sociales de la comunidad que construye el territorio de interés.

Quizás, en Colombia el proyecto más reconocido de patrimonio agrario y cultural sea el proyecto del "Paisaje Cultural Cafetero", reconocido en 2006 por la UNESCO. Muchos trabajos se han adelantado para la investigación e implementación de la propuesta. Sin embargo, el tema sensible para este tipo de casos es, como sucede en gran parte de los países, que no existe un marco legal para amparar la protección del paisaje y el patrimonio natural. Con este planteamiento, en el 2010, la Universidad Tecnológica de Pereira - UTP, desarrolló un proyecto para la construcción de una guía para la integración del Plan de Manejo del Paisaje Cultural Cafetero en el Ordenamiento Territorial de los municipios que lo conforman. La idea de esta guía es establecer las estrategias y medidas que se deben aplicar en las localidades para la preservación de los valores patrimoniales del paisaje.

La guía busca aportar al manejo y conservación del Paisaje Cultural Cafetero

- PCC, a través de una orientación para Integrar los objetivos, estrategias y medidas de conservación del Plan de Manejo del PCC en la revisión y ajuste de los planes, esquemas y planes básicos de ordenamiento territorial de los 47 municipios que componen el área delimitada a partir de una metodología participativa (PCC). 
Los autores reflexionan sobre el hecho de que los organismos públicos, en cumplimiento de sus funciones, deben propender porque las políticas de ordenamiento realmente funcionen como el punto de encuentro entre las políticas sectoriales locales, de manera que pueda lograrse la coordinación y armonía necesarias para que los modelos de ordenamiento, que implican la integración de varias localidades puedan desarrollarse. (Universidad Tecnológica de Pereira, 2010). Para la formulación de la guía se cumplieron las siguientes etapas: un diagnóstico del paisaje, que sirve como base para la segunda etapa de construcción de programas y proyectos $y$, finalmente, una propuesta para la articulación con los planes de ordenamiento territorial. El documento indica de manera práctica el proceso lógico de articulación a partir de un modelo de integración.

\section{Las unidades productivas agropecuarias (UPA) en la interpretación del territorio}

Como marco de análisis de este estudio se propone la unidad productiva agropecuaria (UPA) en relación con las tierras de las que derivan su sustento las familias campesinas. La Ley 160 de 1994 identifica más claramente este espacio productivo como una unidad agrícola familiar (UAF)

Empresa básica de producción agrícola, pecuaria, acuícola o forestal, cuya extensión, conforme a las condiciones agroecológicas de la zona y con tecnología adecuada, permite a la familia remunerar su trabajo y disponer de un excedente capitalizable que coadyuve a la formación de su patrimonio (INCODER, 20I3).

El concepto normativo del INCODER hace evidente el rol productivo y económico de las UAF; sin embargo, esta es una visión unidimensional y limitada de la complejidad del espacio vital de las comunidades campesinas. Las UPA, de las que aquí en adelante se hablará, están más relacionadas con la idea de la "finca", siendo la unidad de expresión rural compuesta por los factores socioculturales y económico-productivos del desarrollo familiar que interactúan con el espacio geográfico que habitan para dotarlo de sentido social. Es un espacio decididamente complejo y dinámico -para nada es estático- que genera una huella en el uso de los recursos naturales y en su relación con las demás fincas que comparten la localidad. Así, estas unidades recogen el concepto de territorio como "sistema de configuraciones de objetos materiales y sociales mediados por relaciones sociales que modifican y transforman la naturaleza lo cual implica su inseparabilidad" (Santos, 1996) y demuestran su validez en la construcción del paisaje, para el caso particular de este trabajo, del paisaje agrario.

\section{HACIA LA IDENTIFICACIÓN DE LAS CARACTERÍSTICAS PAISAIÍSTICAS NATURALES DEL MUNICIPIO DE ONZAGA}

Las experiencias referidas anteriormente sientan una serie de precedentes para el caso particular del municipio de Onzaga. Por tanto, el punto de partida para la lectura del territorio rural en cuestión es el análisis de las unidades productivas agropecuarias, ya como respuesta lógica a la principal orientación económico-productiva de la población rural del municipio en mención. En este contexto, la investigación como proyecto pretende responder a la pregunta: ¿Cuáles son las características técnicas y socioeconómicas de las unidades productivas en el municipio de Onzaga como expresiones del paisaje agrario local, en función de su aplicación en el ordenamiento territorial? La respuesta a este cuestionamiento fundamentará la definición de una serie de herramientas metodológicas para la identificación y análisis de las características del paisaje, haciendo foco en esta ocasión en el paisaje agrario del municipio de Onzaga, utilizando las fincas como unidad 
de análisis, de manera que se ofrezca un diagnóstico inicial que apoye la determinación del papel que puede llegar a cumplir este como herramienta para la planificación y la gestión de territorios con vocación agraria.

Para tal fin, la lectura del territorio inicia a partir de una visualización a escala amplia del territorio donde se tienen en cuenta los subsistemas que lo conforman, entre estos el medio físico o natural, la infraestructura, la población y el poblamiento. En líneas generales, la primera aproximación lleva a ubicar el municipio de Onzaga en el territorio nacional. Este está localizado al sur oriente del departamento de Santander, sobre la cordillera Oriental colombiana y en la cuenca media del río Chicamocha. Es un municipio con alturas entre 900 y 3.000 msnm que representan su variedad de climas y de topografía; el $35 \%$ del área se encuentra en clima medio en alturas menores de 2.000 msnm y la gran mayoría del territorio, el $65 \%$ se ubica en clima frío en alturas correspondientes con el piso térmico del subpáramo mayores a $2.000 \mathrm{msnm}$ y menores a $3.000 \mathrm{msnm}$.

La extensión total del municipio es de $486.76 \mathrm{~km}^{2}$, de los que solo $0.4031 \mathrm{~km}^{2}(0,08 \%)$ corresponden al área urbana y los restantes $486.3399 \mathrm{Km}^{2}(99,9 \%)$ están en territorio rural. La población total es de 5.122 habitantes de los cuales el 79\%, 5003 habitantes, se ubican en el sector rural (Alcaldía Municipal de Onzaga, 2016). La economía local se basa en alto porcentaje en la agricultura familiar a pequeña escala o economía campesina. En la actualidad el municipio dedica un 3,64\% ( 1.773 hectáreas) de sus tierras a los cultivos agrícolas, destacándose el fique, maíz, la caña panelera, el frijol, la yuca, el café y los frutales como sus principales cultivos. En el sector pecuario, la ocupación del territorio alcanza el 30,83\% ( I5.0 I I ha). El territorio restante corresponde a zonas boscosas andinas y subandinas en una extensión aproximada de 26.783 ha y 5.063 ha en bosques de páramo (Alcaldía Municipal de Onzaga, 2016).

El principal acceso desde la capital del departamento de Santander se hace por la vía Bucaramanga - San Gil (97km), San Gil - Mogotes - San Joaquín - Onzaga (82km). Onzaga cuenta con $219 \mathrm{~km}$ de vías carreteables que la comunican con los municipios aledaños y colindantes: al norte Molagavita; el río Chicamocha al medio; al Oriente Cobarachía, Subachoque, Soatá y Sativa (Boyacá); al sur, Sativa Norte y por el occidente con San Joaquín y Coromoro (figura 4).

Una de las principales características ambientales del municipio es su riqueza hídrica. El río Onzaga y la Quebrada de Los Micos, ubicada en el corregimiento de Padua, son las principales fuentes hídricas municipales. El municipio se integra a la cuenca del río Chicamocha que allíse subdivide en dos subcuencas: la del río Onzaga, con aproximadamente el $78 \%$ del área y seis microcuencas que la alimentan y la de la quebrada Los Micos con el $22 \%$ del área restante. Todas las aguas finalmente corren de sur a norte para desembocar en el río Chicamocha. En relación con el clima, el conjunto de los factores meteorológicos de temperatura, vientos, precipitación, humedad relativa, entre otros, no es posible tener datos muy precisos en razón a la falta de continuidad y actualización de la información recolectada por el IDEAM.

De lo anteriormente expuesto, queda clara la vocación agrícola y pecuaria del municipio de Onzaga, por lo que la investigación determinó en forma más que natural emprender un análisis de las características socioeconómicas y productivas de las fincas en Onzaga, tomando una segunda escala de análisis que exigía mirar el territorio a partir de su división política municipal, para establecer puntos de reflexión y de análisis particularizado con respecto de las características socioeconómicas y productivas, por lo que se opta por un número de 60 fincas, seleccionadas a partir de su distribución sobre las veredas del municipio (figura 5) y la caracterización del territorio en el cual se insertan. 
Figura 4. Localización del municipio de Onzaga y su sistema vial

Fuente: Elaboración propia a partir del EOT del municipio

Figura 5. División político-administrativa de Onzaga, Santander

Fuente: Elaboración propia a partir del EOT del municipio
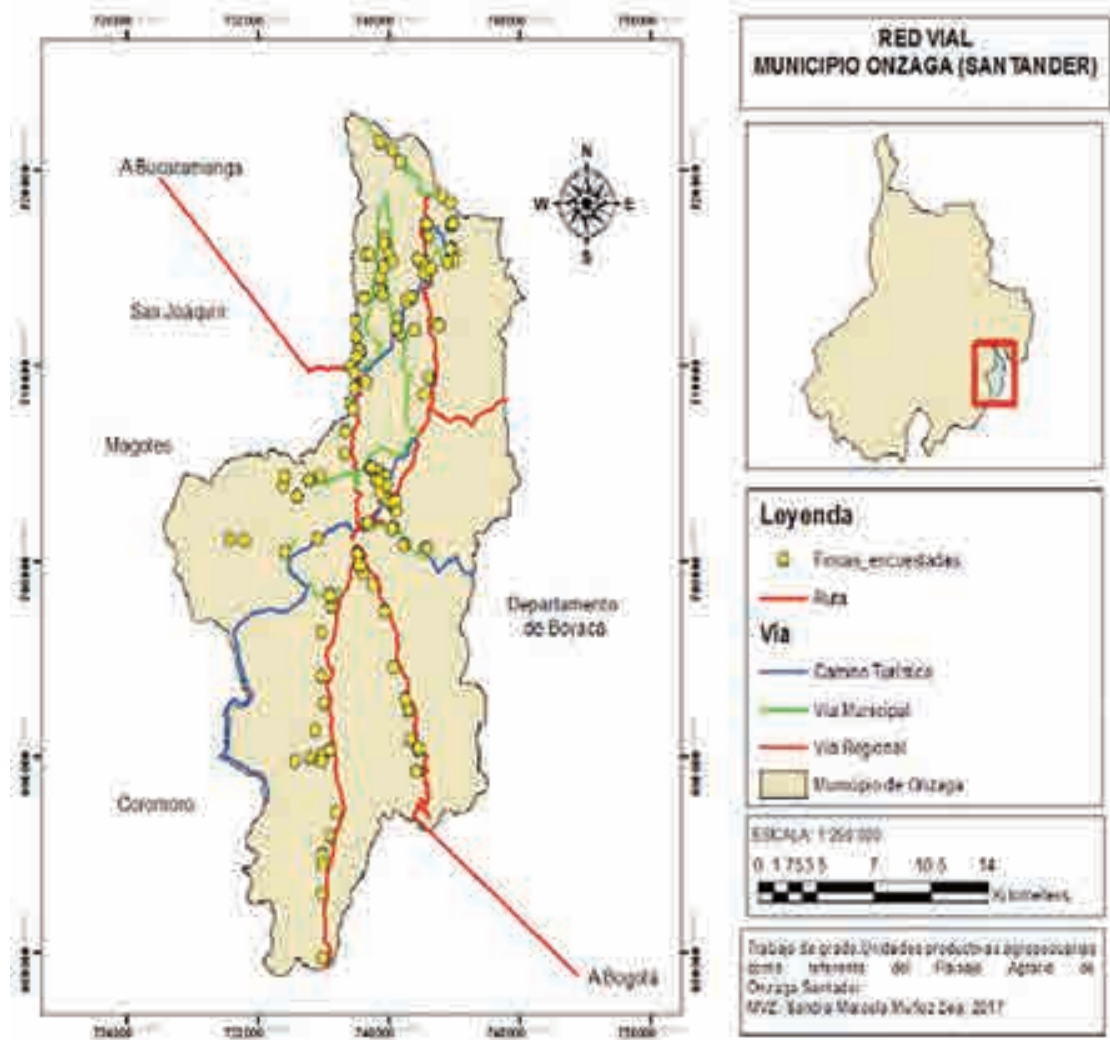

Loyenda

d Toarecuestit.

- ana

Via

Cones talse.

- Vivanipal

- varpegeas

Denapsatonew.

cacestantion

$0.17558 \quad 2 \quad 105 \quad 4$

50

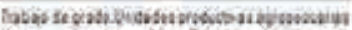

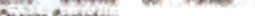

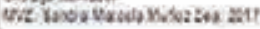

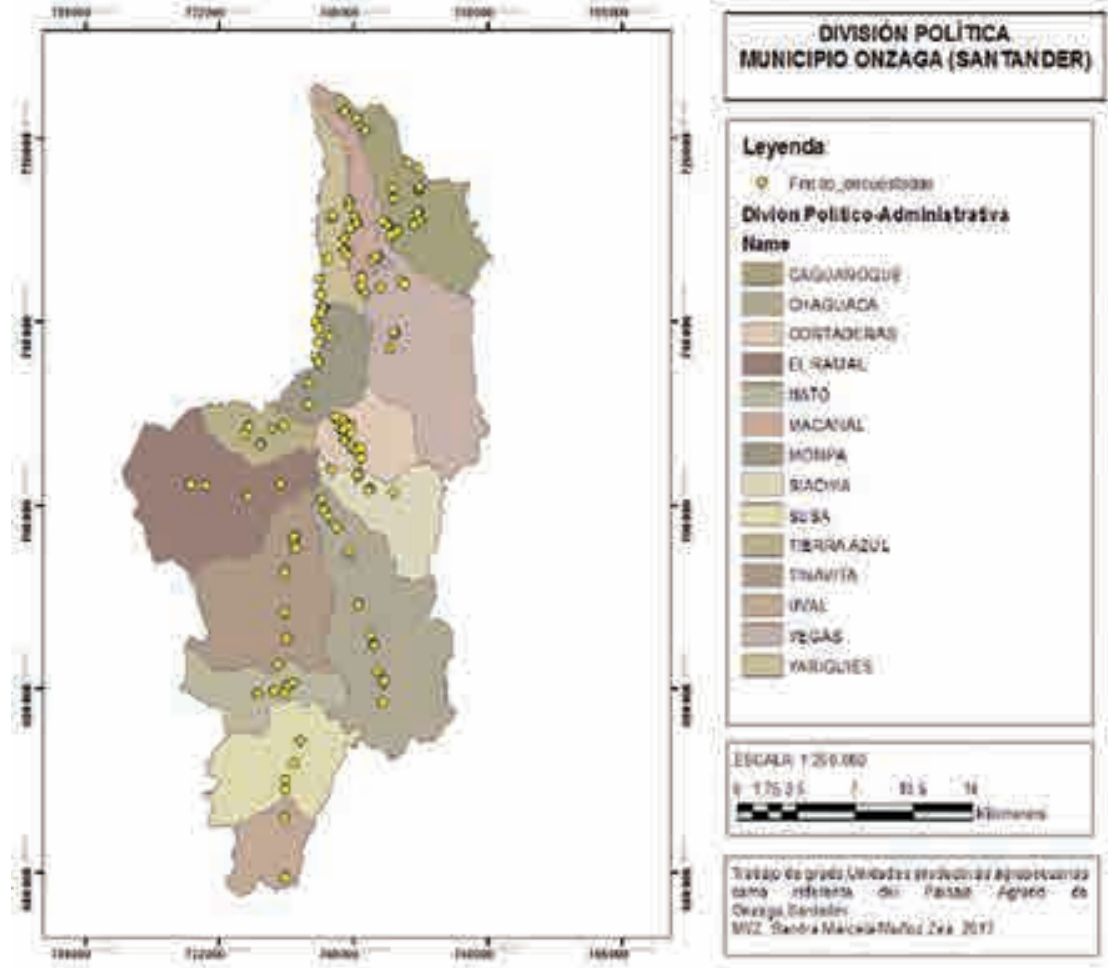


Es este un punto crucial de la investigación que también pone el límite al alcance del presente artículo. En síntesis, se identifica un municipio que se divide en 14 veredas integradas por 27 sectores, división que responde más a factores sociales que de otra índole, visiblemente conectados a la llegada de proyectos productivos que implican divisiones territoriales y al mismo tiempo cambios en las características productivas, sociales y económicas de los productores agropecuarios especialmente. Las divisiones se dan también por situaciones de acceso al agua, o a otros recursos naturales y materiales. A su vez, se identifican una serie de "fincas" que serán asumidas como unidades productivas agropecuarias - UPA, las cuales serán por tanto sometidas a un proceso de estudio que se fundamenta en las bases teóricas expuestas al inicio del artículo, así como en los estudios de caso que se enunciarán más adelante. Este será el apartado de la investigación que llevará a un proceso de confrontación entre la realidad territorial, los postulados teóricos y la normativa existente y cuyos resultados hacen parte de un posterior informe debido a su extensión y complejidad.

\section{CONCLUSIONES}

Colombia, especialmente en las condiciones que en la actualidad el Proceso de Paz exige para los modelos de desarrollo, entiende la importancia que el contexto rural juega en este proceso y en la superación de buena parte de las dificultades que se dan en el país para el desarrollo equitativo. Sin embargo, los instrumentos construidos para la planificación del desarrollo no están cumpliendo al menos con dos exigencias mínimas: que respondan a las dinámicas y características particulares de los territorios de manera que se garantice su pertinencia y, que cumplan con la función previsiva de la planificación por encima del mero cumplimiento normativo de su formulación.

El aprovechamiento del análisis del paisaje como herramienta para el diagnóstico de un territorio ha demostrado su validez como primer elemento de la planificación y base de la estructura sólida de este proceso, premisa corroborada gracias a los resultados de varias experiencias llevadas a cabo en el ámbito internacional, algunas de ellas expuestas en este artículo. La aplicación de esta herramienta en el contexto colombiano, territorio con una disponibilidad estratégica de recursos ambientales $y$ una dinámica rural histórica con un renovado interés en el futuro del actual proceso de paz colombiano, sería sin duda una decisión afortunada en miras de cumplir con las exigencias vitales de la planificación para el desarrollo.

La relación de las UPA presentes en el territorio con el paisaje natural y agrario es tan evidente como se ha justificado en el desarrollo de este documento. Por esta razón la fusión en el paisaje de las técnicas de producción, la salud de los ecosistemas, la eficiencia productiva de las actividades económicas, la relación de la población con el uso de los recursos naturales, el acervo del patrimonio cultural productivo, el bienestar social de la comunidad y la eficiencia de las políticas de desarrollo territoriales justifican el aprovechamiento de las UPA como referente para la planificación del territorio. A partir de la teoría existente y de los estudios de caso revisados, emerge claramente la pertinencia del uso de las UPA como unidad de análisis para el diagnóstico territorial, siempre teniendo en cuenta una lectura paisajística, es decir, en el marco de la complejidad y la multidimensionalidad que alberga su funcionamiento.

También queda claro que ni la normativa, ni las capacidades técnicas de las localidades facilitan la tarea. En el país la normativa actual no ampara de manera clara y directa la protección y el manejo sostenible del paisaje, por lo que tampoco identifica 
su rol en el ordenamiento del territorio. Esto se conjuga con que en las localidades es común que los funcionarios encargados desconozcan la teoría que sustenta la aplicación de la planificación en el ordenamiento, y que de igual forma ignoren la importancia del uso de herramientas adecuadas y consideren los diagnósticos como una labor de menor rango.

Por todo ello, se espera que la parte conceptual de este trabajo, más allá de la lectura de la realidad territorial del municipio de Onzaga, sea de utilidad para la lectura del territorio de eventuales experiencias similares y particularmente para considerar una revisión de la instrumentalización normativa y de otras herramientas análogas, de manera que se afiance su utilidad, se popularice su uso y conocimiento, se supere el desarrollo de la planificación como el simple cumplimiento de una norma y se invite a la participación de entidades académicas en el proceso, las cuales le otorguen un nuevo sentido teórico a esta labor.

\section{REFERENCIAS}

Agudelo, L. C. (2006). La ruralidad en el Ordenamiento Territorial en Colombia. Centro de Estudios en Planificación, Políticas Públicas e Investigación Ambiental. Recuperado de http://www.ceppia.com.co/Documentos-tematicos/TERRITORIAL/ LA-RURALIDAD-EN-EL-ORDENAMIENTO-TERRITORIAL-EN-COLOMBIA.pdf

Alcaldía Municipal de Onzaga. (2000). Esquema de Ordenamiento Territorial. Municipio de Onzaga Santander. Onzaga: Alcaldía de Onzaga.

Alcaldía Municipal de Onzaga. (2016). Plan de Desarrollo Territorial 2016-2019. Onzaga, Santander: Alcaldía Municipal.

Arriola, I. G. (20I0). El plan de manejo para el Paisaje Agavero y las Antiguas Instalaciones Industriales de Tequila. Universidad Javeriana. Recuperado de http://revistas. javeriana.edu.co/index.php/revApuntesArq/article/view/8933/7219

Cano, N. (2006). Paisaje y desarrollo rural sostenible en Euskadi: la importancia de su ordenación y gestión. Euskomedia. Kultura Topagunea. Recuperado el de http://www. euskomedia.org/PDFAnlt/congresos/16/16129/35.pdf

Castillo, J., \& Martínez, C. (20I4). El patrimonio agrario: definición, caracgerización y representatividad en el ámbito de la UNESCO. Universidad de la Rioja. Recuperado de https://dialnet.unirioja.es/descarga/articulo/4903360.pdf

Convention Européenne du Paysage. (2000). Convention européenne du paysage. Conseil de l'Europe. Recuperado el de http://www.coe.int/t/dg4/cultureheritage/ heritage/Landscape/default_fr.asp

Cortijo, J. (2013). Paisaje y patrimonio rural: Las "mieses" en Valdáliga, Herrerías y Rionansa. Universidad de Cantabria. Recuperado de https://repositorio.unican.es/ xmlui/handle/ 10902/3892

Echeverri, R. (20II). Reflexiones sobre lo rural: economía rural, economía de territorios. En Hacia una nueva definición de "rural" con fines estadísticos en América Latina. CEPAL. Santiago.

Gómez, A. (1997). El paisaje agrario desde la perspectiva de la ecología. Universidad de Alcalá. Recuperado de https://portal.uah.es/portal/page/portal/epd2_profesores/ prof I 2 1902/docencia/5AE05 I8BC52452A8E0440003BAB IA89D 
Gómez, A. (20/2). El paisaje y su consideración en la planificación del desarrollo y el ordenamiento territorial. Aplicación al PDOT del cantón de Cuenca. Universidad Verdad. Ordenamiento Territorial, 57. Revista de la Universidad del Azuay. Recuperado de http://www.uazuay.edu.ec/bibliotecas/publicaciones/UV-57.pdf

Gómez, D., Gómez, A., \& Gómez, M. (20I2). El paisaje. Análisis, diagnóstico y metodología para insertarlo en la formulación de planes y proyectos. Madrid.

Gómez, J., \& Riesco, P. (2010). Marco conceptual y metodológico para los paisajes españoles. Aplicación a tres escalas espaciales. Dialnet. Recuperado de http://dialnet. unirioja.es/servlet/libro?codigo $=4609 \mid 4$

INCODER. (2013). Determinación de las Unidades Agrícolas Familiares (UAF) para el departamento del Cauca. Pontificia Universidad Javeriana. Recuperado de https://www. javerianacali.edu.co/sites/ujc/files/JaverianaFiles/informe_cauca_version_sept_I4.pdf

Irastrorza, P. (2006). Integración de la ecología del paisaje en la planificación territorial. Aplicación a la Comunidad de Madrid. Archivo Digital Universidad Politécnica de Madrid. Recuperado de http://oa.upm.es/468/I/PEDRO_IRASTORZA_VACA.pdf

Martín, V. O. (2000). Aproximación tipológica a los paisajes agrarios actuales de Canarias. Universidad de La Laguna. Recuperado de https://dialnet.unirioja.es/servlet/ articulo?codigo $=105627$

Mata, R. (2008). El paisaje, patrimonio y recurso para el desarrollo territorial sostenible. Conocimiento y acción pública. Universidad de Córdoba. Recuperado de http:// www.uco.es/ gt l tomam/master/paisaje/mata.pdf

Minambiente. (2004). Guía metodológica para la implementación del expediente municipal. Ministerio de Ambiente, Vivienda y Desarrollo Territorial de Colombia. Recuperado de http://www.metropol.gov.co/observatorio/Expedientes\%20Municipales/Documentos\%20tecnicos/Guia_Expediente_Municipal.pdf

Ministerio de Cultura. (20II). Paisaje Cultural Cafetero. Un paisaje cultural productivo en permanente desarrollo. Paisaje Cultural Cafetero. Recuperado de http:// paisajeculturalcafetero.org.co/static/files/cartillaministerio.pdf.pdf

Municipio de Onzaga. (2000). Esquema de Ordenamiento Territorial. Municipio de Onzaga, Santander. Recuperado de http://cdim.esap.edu.co/BancoMedios/Documentos\%20PDF/onzagasantandereotdr2000.pdf

Municipio de Onzaga. (2016). Plan de Desarrollo 2016 - 2019 "Digale Sí a Onzaga". Municipio de Onzaga. Recuperado de www.onzaga-santander.gov.co

Santos, M. (1996). O Retorno do Território. En Milton Santos, Maria A. A. de Souza y María L. Silveira. (Coords.). Território: Globalização e Fragmentação (pp. I5-20). São Paulo: Hucitec-ANPUR.

Silva, R. (2008). Hacia una valoración patrimonial de la agricultura. Revista Electrónica de Geografía y Ciencias Sociales. Recuperado de http://www.ub.edu/geocrit/ sn/sn-275.htm

Universidad Tecnológica de Pereira. (2010). Guía para la integración del Plan de Manejo del Paisaje Cultural Cafetero en el Ordenamiento Territorial. Universidad Tecnológica de Pereira. Colombia. Recuperado de http://www.cgpcc.edu.co/assets/ guiaordenamientoterritorial.pdf

M 97 REVISTA M VOL. 14 ENERO - DICIEMBRE 2017 • FACULTAD DE ARQUITECTURA • UNIVERSIDAD SANTO TOMÁS COLOMBIA 
Zoido, F. (1999). El paisaje y su utilidad para la ordenación del territorio. Centro de Estudios. Paisaje y Territorio. Recuperado de http://paisajeyterritorio.es/assets/ el-paisaje-y-su-utilidad-para-la-ordenacion-del-territorio.-zoido-naranjo\%2C-f.pdf 